\title{
Hybrid Paraplegic Locomotion with the ParaWalker Using Intramuscular Stimulation: A Single Subject Study
}

\author{
A. V. Nene, MB, MS, MChOrth, S. J. Jennings, BSc \\ The Orthotic Research and Locomotor Assessment Unit, The Robert fones E Agnes \\ Hunt Orthopaedic Hospital, Oswestry, Shropshire, SY10 7 AG, UK.
}

\begin{abstract}
Summary
Functional intramuscular electrical stimulation of the gluteus maximus and gluteus medius on the stance side whilst walking is reported. The subject was an adult, male, post-traumatic complete paraplegic with lesion level at T7. He had been using surface stimulation to aid his walking with the adult ParaWalker Orthosis but suffered unpleasant anterior abdominal wall muscle contractions with high amplitude stimulation. The experimental use of percutaneous intramuscular Platinum/Iridium wire electrodes demonstrated that more forceful contractions of the buttock muscles could be achieved without any effect on abdominal wall musculature. This prompted the use of a permanent, implanted system. The system is inductively coupled to an external control device and is working satisfactorily 7 months after implantation.
\end{abstract}

Key words: Paraplegia; Intramuscular-FES; Implant; Hybrid; ParaWalker.

\section{Introduction}

The ParaWalker was initially developed at ORLAU to allow reciprocal gait ambulation in paraplegic children, with the aid of crutches. It relies on lateral stiffness of the leg member to prevent adduction but when used for adults some adduction cannot be prevented and an extra burden is put on the supporting arms (Nene and Major, 1987). Gluteal muscle contraction brought about by surface electrical stimulation has been shown to assist ambulation (Nene and Andrews, 1986; McClelland et al., 1987). The strength of gluteal muscle contraction using surface electrodes is limited in many patients because concurrent anterior abdominal wall muscle contraction occurs if the stimulus is too great. It was suggested that this was due to depolarisation of muscle afferent axons within the nerve fibres (Nene and Andrews, 1986). Another possibility is that it is a manifestation of a cutaneous, withdrawal reflex and that it could be avoided by stimulation of the inferior and superior gluteal nerves using intramuscular electrodes. The inferior gluteal nerve supplies gluteus maximus and the superior gluteal nerve supplies gluteus medius; the main muscles of the group. 
An implant and transmission sytem has been developed at the Medical Research Council's Neurological Prosthesis Unit, London, which will tolerate movement between the transmitter and receiver. The two isolated outputs of the receiver can be controlled independently for amplitude (Donaldson, 1987a, b, c; Ivall, 1987). Two such receivers, one on each side, with electrodes sited in gluteus maximus and gluteus medius appeared to meet the requirements of some ParaWalker users and the first trial of their use for a paraplegic subject is described here.

Prior to the implantation, an experiment using percutaneous, wire electrodes was performed to see whether forceful gluteal contraction could be achieved without anterior abdominal wall muscle contraction.

\section{Subject}

The subject was a 29-year-old male, traumatic, complete paraplegic with a lesion at level T7. He was $1.74 \mathrm{~m}$ tall and weighed $63.6 \mathrm{~kg}$ with his orthosis. He was a proficient user of the ParaWalker which had been supplied 3 years previously. Over a period of 11 months his gluteal muscles had been retrained using the Princess Margaret Rose Orthopaedic Hospital, SMS 2 surface stimulator with a pair of surface electrodes (Myocare, 3M) positioned to stimulate both gluteus maximus and gluteus medius on each side. The subject also used the surface stimulator whilst walking. Some reduction in the vertical crutch impulse was shown whilst walking but any further reduction could not be achieved because of his inability to increase the amplitude of stimulus strength without anterior abdominal wall muscle contractions.

\section{Muscle stimulation by percutaneous electrodes}

Percutaneous electrodes were made from strands of $80^{\circ}{ }_{0} \mathrm{Pt} / 20^{\circ}$ o Ir wire (diameter $0.07 \mathrm{~mm}$ ) coated in Trimel (Polyimide) and supported in a $125 \mathrm{~mm}$, $18 \mathrm{G}$ spinal needle. The cathode consisted of 2 strands. The end $1 \mathrm{~cm}$ of each strand was bent back to form barbs. These ends were stripped bare of Trimel by heating in a flame, exposing a total geometric area of approximately $4 \mathrm{~mm}^{2}$. The anode was made from 4 strands. The end $2 \mathrm{~cm}$ of each was bent back and a total, geometric area of approximately $17 \mathrm{~mm}^{2}$ was exposed. The minimum area of cathode and anode required to avoid electrolysis or corrosion was calculated from consideration of the electrical pulse to be delivered (Craggs et al., 1986; Donaldson and Donaldson, 1986).

The motor points for the gluteus maximus and gluteus medius were located on the skin surface using the surface stimulator and marked. Under sterile conditions the cathodes were introduced through $75 \mathrm{~mm}, 11 \mathrm{G}$ cannulae inserted at the marked sites to a depth approximately $2 \mathrm{~cm}$ away from the underlying bone (Fig. 1). The cannulae and needles were removed leaving the electrodes in place. Anodic electrodes were introduced in the same way at convenient sites approximately $3 \mathrm{~cm}$ away from the corresponding cathodes.

Returning to the Gait Laboratory and with the subject wearing his ParaWalker, the distal ends of the electrode wires were connected to the percutaneous stimulator system. Pulses of duration $0.2 \mathrm{~ms}$, amplitude adjustable up 


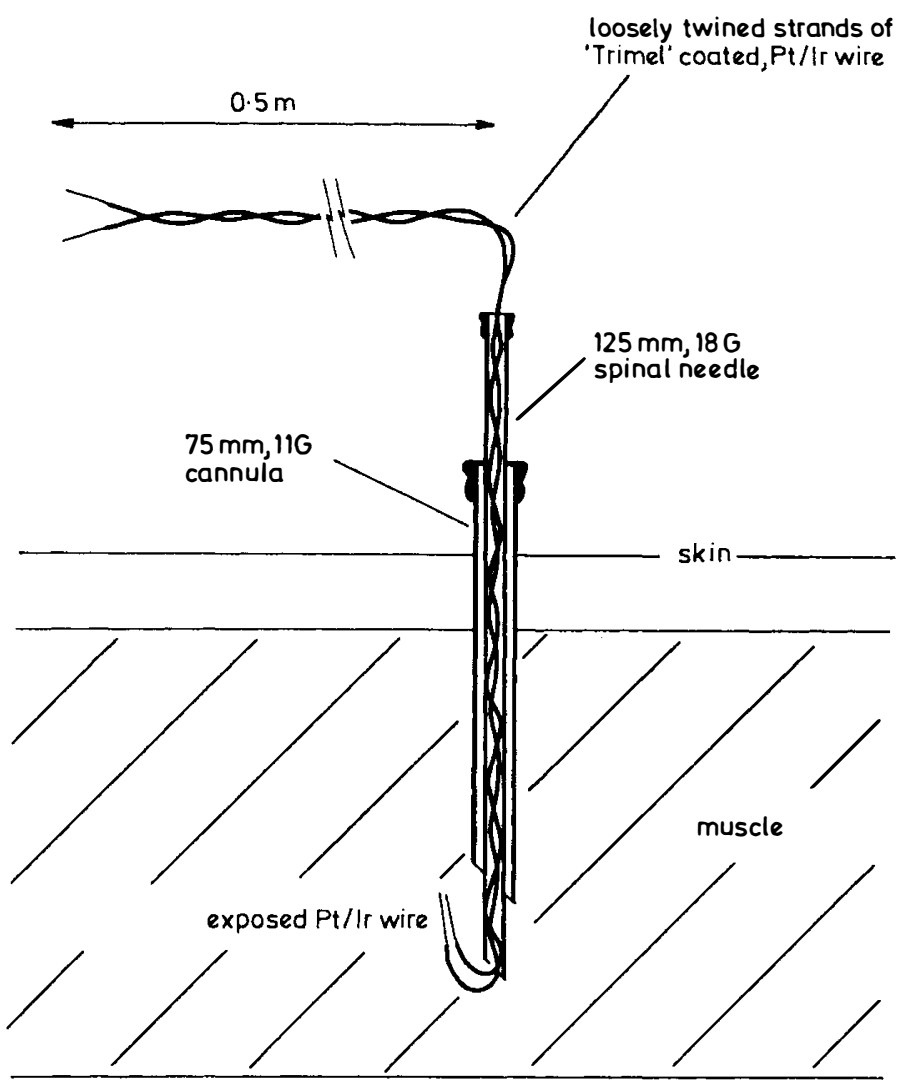

Figure 1 Schematic diagram of percutaneous electrode insertion apparatus.

to $20 \mathrm{~V}$, at a repetition rate of 25 pulses per second, were provided via an inductively coupled isolator for each pair of electrodes. The cathodes were coupled using a series 1 micro-Farad capacitor to ensure no net flow of charge. The amplitude of the stimulus could be adjusted independently for each of the four muscles.

With percutaneous electrodes more forceful contractions of gluteus maximus could be achieved, than with surface stimulation. Gluteus medius could also be made to contract with reasonable force. No cross stimulation between muscles was observed. The anterior abdominal wall was palpated and no muscle contraction was felt at any level of stimulation. The subject reported no discomfort. This is in agreement with the hypothesis that the abdominal wall muscle contraction is mediated by a cutaneous reflex.

Stimulation of the stance side gluteii were coordinated by the subject using a finger switch on the contralateral crutch whilst walking in the ParaWalker. The subject felt that walking was easier when his gluteal muscles were stimulated with percutaneous electrodes than with surface electrodes. This suggested that the subject would benefit from a permanent implant system.

After approximately 2 hours of standing and walking and approximately 4 hours after insertion the electrodes were removed. They all emerged cleanly without breaking. 


\section{Muscle stimulation by implanted electrodes}

The implant consists of left and right receivers (diameter $40 \mathrm{~mm} \times 15 \mathrm{~mm}$ ) each with two outputs, sited over the lower costal surfaces. Each anodic and cathodic pair of electrodes is formed from the distal end of a Cooper cable (triple helix of $\mathrm{Pt} / \mathrm{Ir}, 0.07 \mathrm{~mm}$ diameter wire coated in silicone with an outer diameter of $2 \mathrm{~mm}$ ). The proximal end is connected to the receiver outputs. The cables run subcutaneously and conduct the charge to the electrodes positioned in the gluteal muscles. Each of the capacitively coupled receiver outputs can deliver a regulated charge of up to 2 micro-Coulombs during a pulse of approximately $0.1 \mathrm{~ms}$. at the rate of 25 pulses per second. Details of a similar receiver are given elsewhere (Donaldson, 1987c). The external equipment consists of an oscillator/transmitter $(70 \times 105 \times 10 \mathrm{~mm})$ fixed to the skin over each receiver and a control box $(60 \times 110 \times 190 \mathrm{~mm}, 0.8 \mathrm{~kg})$ attached to the orthosis and operated by a finger switch on each crutch handle. The $3 \mathrm{MHz}$ transmission system achieves insensitivity to coil separation within a $45 \mathrm{~mm}$ radius (Donaldson, 1987a, b; Ivall 1987) and the whole system is powered by 2 pp3 Ni-Cad batteries which last approximately 1 hour between charges.

The system was implanted surgically with antibiotic prophylaxis. No anaesthesia was necessary since all incisions were below the neurological level. Under strict sterile conditions an incision, approximately $50 \mathrm{~mm}$ long, was made over the gluteal region between previously marked motor points for gluteus maximus and gluteus medius. The incision was at an angle of approximately $45^{\circ}$ to the mid axillary line. A probe, connected to a battery powered stimulator, was inserted via a ' $U$ ' shaped cannula. The stimulator, coupled through a 100 microFarad capacitor, provided continuous pulses at a rate of 3 pulses per second, each of $0.35 \mathrm{~ms}$ duration and amplitude adjustable to $20 \mathrm{~V}$. The best sites for stimulation within the muscles were found using the probe as cathode and the self-retaining metal retractor, used to keep the incision open, as anode.

After confirming the sites, the distal ends of the electrode cables were inserted via the cannula. The proximal ends were then routed subcutaneously to a second, $40 \mathrm{~mm}$ long incision made laterally, perpendicular to the mid axillary line and mid way between the lower costal margin and the iliac crest. From there, they were routed, again subcutaneously, to a third incision on the lower costal surface. The proximal ends of the cables were connected to the receiver which was then accommodated in a subcutaneous pouch prepared at the incision site. All the wounds were closed after careful haemostasis. The procedure was repeated for the opposite side.

Training of the gluteal muscles was discontinued to allow time for the electrodes to become securely fixed to the surrounding tissue.

The first post-operative visit was made at 6 weeks. The subject's wounds were inspected and found to have healed satisfactorily. Each muscle could be made to contract with activation of the appropriate channel and no cross stimulation between muscles was observed. The weakness of contraction observed on this occasion may have been due to atrophy of the unexercised muscle and the subject restarted his muscle training programme using the surface stimulator.

A second post-operative visit was made at 4 months. Contractions of gluteus maximus and gluteus medius were more forceful than the previous visit. They 


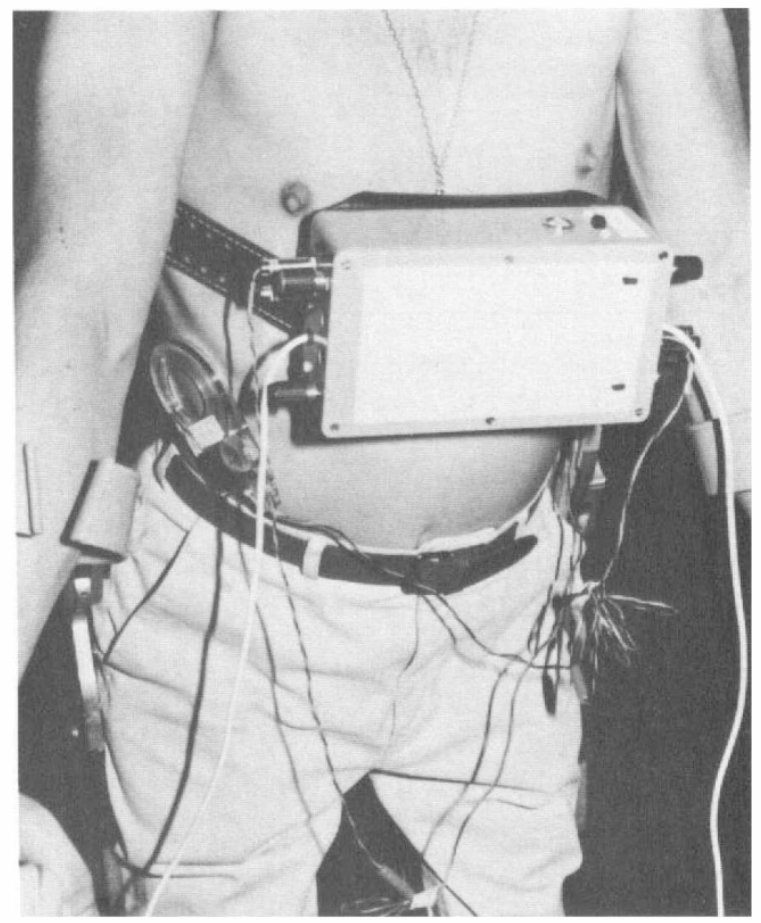

Figure 2 The implant system in situ showing the control box and the right hand side oscillator/transmitter.

were less forceful than when using percutaneous or surface electrodes but no abdominal wall muscle contraction occurred. Good differentiation between gluteus maximus and gluteus medius was maintainted on the left side but some cross stimulation was observed on the right. Functional stimulation was begun using the implant system. Each oscillator/transmitter was fixed, using Dow Corning medical adhesive, with the transmitter coil centred over the protrusion of the receiver on the lower costal surface. The control box was fixed to the ParaWalker. The subject activated stimulation from the finger switches on the crutch handles whilst walking (Fig. 2).

On returning home the subject continued to exercise his gluteii using the surface stimulator and to use the implant system whilst walking.

At $6 \frac{1}{2}$ months a third post-operative visit was made by the subject to the laboratory. It was determined that the implant was working satisfactorily, still without abdominal wall muscle contraction, as on the previous visit. On this occasion measurements of crutch impulse, speed, and cadence were made.

While the subject walked along a $6.15 \mathrm{~m}$ path, the number of foot falls and the time taken were recorded and used to evaluate speed and cadence. At the same time the vertical force applied through the left or right crutch was recorded from a force platform (Kistler, 9261B) embedded in the middle of the path. This was used to derive crutch impulse (the integral of force with respect to time). Twenty walks were recorded with the subject resting in the standing position for 1 minute after each walk. Pairs of walks with left and right impulse measurements were recorded with and without electrical stimulation, alternately. 
Table Impulse, speed and cadence without and with electrical stimulation by means of the implant system.

\begin{tabular}{lcc}
\hline & Without & With \\
\hline Right crutch impulse (N.s.) & $163(20)$ & $156(37)$ \\
Left crutch impulse (N.s.) & $184(36)$ & $159(16)$ \\
Speed (m.s ${ }^{-1}$ ) & $0.36(0.03)$ & $0.39(0.04)$ \\
Cadence (steps.min $^{-1}$ ) & $49.6(2.0)$ & $52.6(2.8)$ \\
\hline
\end{tabular}

Mean values are presented with values of standard deviation in brackets.

The results are shown in the Table and indicate that the use of electrical stimulation leads to some decrease in the mean value of crutch impulse concurrently with an increase in speed and cadence but the differences are small compared with the variation in the measurements.

\section{Discussion}

Gluteal muscle contraction achieved using the implant system was not as good as when using percutaneous electrodes. The electrical characteristics of the stimulus used in the two methods were not identical and this may account for the different results. The electronic circuit which allows independent control of the amplitudes of two outputs from each implanted receiver limits the charge transfer to approximately 2 micro-Coulombs. In contrast, the percutaneous sytem was capable of delivering a charge of up to 25 micro-Coulombs (assuming a tissue resistance of $390 \mathrm{ohms}$ ). Subjective observation would suggest that the ratio of strengths of stimuli to the inferior and superior gluteal nerves is not critical. The use of receivers each with two outputs in fixed ratio would allow greater charge to be delivered.

In a uniform medium, reducing the distance between cathode and anode would produce greater charge concentration for a given current but a smaller volume would be affected. This would require greater precision in locating the electrodes around the motor point within the muscle. The cathode to anode spacing used in the implanted system may not have been the best compromise.

In locating the electrode site a cathodic probe was inserted into the muscle, with the metal retractor as anode at the skin surface. Under these circumstances a relatively large volume of tissue is affected by the flow of charge and this may give a misleading impression of where to site the closely spaced cathode and anode at the distal end of the Cooper cable.

Limited improvement in the walking ability was shown in this subject, using the implanted intramuscular electrical stimulation system. Possible reasons and their solutions have been described above. This subject normally walks well and does not put a great adducting moment on the orthosis but a heavier, taller subject may be expected to derive a greater benefit. 


\section{Safety aspects}

\section{Mechanical}

Fail safe. Since the electrical stimulation system is used in conjunction with the mechanical orthosis (ParaWalker) it is unlikely that the subject would collapse in case of failure of the electrical system.

The adhesive. The transmitter is adhered to the skin surface using the medical adhesive (Dow Corning) which has been in use for years. It has been proved to be reliable and non toxic over this period. No other adhesive should be used.

\section{Electrical}

Interference. The receiver requires a strictly controlled radio frequency (r.f.) pulse shape in order to send electrical impulses to the muscles. It is unlikely that any other apparatus would match the coding characteristics of this apparatus. The capacity to receive and decode the r.f. signal is distance specific. Unless the transmitter is within a few centimeters of the receiver it will not be able to operate.

Electric shock. The control box uses rechargeable PP3 size batteries. These have to be removed for recharging and so it is impossible for the patient to receive a shock through the mains electricity.

\section{Physiological}

Warming up actively. The subject is asked to perform passive flexion/ extension of the lower limbs and warming up exercises before commencing use of the electrical stimulation. This is to avoid any risk of strains and sprains which may be caused by sudden muscular contraction.

Skin care. The subject is asked to inspect the skin surface under the transmitters after each use. The use of the apparatus should be avoided in the presence of any skin abrasions. Since no current is passed through the skin from the transmitter and the radio frequency energy is very low the authors consider that there is no significant risk of burns.

\section{Conclusion}

It has been demonstrated that, for a paraplegic subject forceful gluteal contractions can be obtained without eliciting detrimental abdominal wall contraction using intramuscular electrodes. This is consistent with the abdominal wall muscle contraction being due to a cutaneous reflex.

\section{References}

Craggs MD, Donaldson NdeN, Donaldson PEK 1986 Performance of platinum stimulating electrodes, mapped on the limit-voltage plane. Part 1, Charge injection in vivo. Medical and Biological Engineering and Computing 24:424-430. 
Donaldson NdEN, Donaldson PEK 1986 Performance of platinum stimulating electrodes mapped on the limit-voltage plane. Part 2, Corrosion in vitro. Mechanical and Biological Engineering and Computing 24:431-438.

DoNALDSON PEK 1987a Power for neurological prostheses. A simple inductive r.f. link with improved performance. Fournal of Biomedical Engineering 9:194-197.

DONALDSON PEK 1987b Three separation-insensitive radiofrequency inductive links. Fournal of Medical Engineering and Technology 11:23-29.

DoNALDSON PEK 1987c The Janus link: two for the price of one? Electronics and Wireless World 93:1097-1098.

IVALL T 1987 Does your coupling coefficient matter? Making inductively-coupled energy less sensitive to coil separation. Electronics and Wireless World 93:577-579.

McClelland M, Andrews BJ, Patrick JH, et al. 1987 Augmentation of the Oswestry ParaWalker orthosis by means of surface electrical stimulation: Gait analysis of three patients. Paraplegia 25:32-38.

Nene AV, ANDREws BJ 1986 An assessment of the ParaWalker hybrid orthosis. Proceedings of Second Vienna International Workshop on Functional Electrostimulation. September: 79-82.

Nene AV, MAJOR RE 1987 Dynamics of reciprocal gait of adult paraplegics using the ParaWalker (Hip Guidance Orthosis). Prosthetics Orthotics International 11:124-127. 